\title{
Human embryonic stem cells create their own niche
}

\author{
Ying $\operatorname{Jin}^{1}$ \\ ${ }^{1}$ Institute of Health Sciences, Shanghai Institutes for Biological Sciences, Chinese Academy of Sciences/Shanghai Jiaotong University \\ School of Medicine, 225 South Chongqing Road, Shanghai 200025, China.yjin@sibs.ac.cn
}

Cell Research (2007) 17:744-745. doi: 10.1038/cr.2007.76; published online 13 September 2007

Experimental evidence demonstrates that the ability of stem cells to self-renew and to differentiate into different types of mature cells depends on both their intrinsic genetic programs and external control from their microenvironment or niche. The concept of stem cell niche was first proposed by Schofield in 1978 to describe a microenvironment that supports stem cells in a mammalian hematopoietic system [1]. Over the last 30 years, more stem cell niches have been identified in the mammalian system, including the hematopoietic stem cell niche in bone marrow, the epithelial stem cell niche in skin, the intestinal stem cell niche, the neural stem cell niche and the germ line stem cell niche in mice [2]). Recently, the concept of stem cell niche is further defined. The niche must have both anatomic and functional dimensions and may be composed of heterologous cell types, extracellular matrix, paracrine factors or non-protein metabolites [3]. More recently, it was shown that disruption in the niche of hematopoietic stem cells leads to the development of myeloproliferative disease [4]. It becomes obvious that a stem cell niche is not static, but dynamic, and can be modified or even created. Although stem cell niche has emerged as critical as stem cell autonomous functions for both our understanding of stem cell biology and the application of stem cells in medicine, a niche for human embryonic stem (hES) cells was not clearly shown until recently Bendall et al demonstrated that IGF and FGF cooperatively establish the regulatory stem cell niche of pluripotent human cells in vitro [5].

hES cells have been derived and cultured on mouse embryonic fibroblast (MEF) cells or conditioned media from MEF (MEF-CM) and in the defined medium without non-human proteins $[6,7]$. Over the last decade there have been great efforts in culture of hES cells and identification of the factors essential to sustaining hES identity. Despite the development of diverse culture systems, the optimal condition for hES cell culture has not been established and hES cells cultured in vitro easily differentiate spontaneously. Therefore, it is particularly important to identify the factors essential for maintaining hES in culture. Using a proteomic screening approach, Bendall et al found that the most prevalent family of growth factors in MEF-CM was insulin-like growth factor II (IGF-II) [5], which is known to play a crucial role in mammalian embryonic development [8]. Furthermore, they detected the receptor for IGF-II, IGF1R in hES cells. The findings suggest that IGF pathway may play an important role in regulating functions of hES cells, along with basic fibroblast growth factor (bFGF), which has been supplemented in all available hES cell culture systems [9]. Although bFGF was predicted to act directly on hES cells since it functions independent of the presence of feeder cells, the IGF pathway had not been investigated in $\mathrm{hES}$ cells. In addition, Bendall et al observed an interesting phenomenon, which is that hES cells cultured in the absence of feeder cells generate Oct-4-expressing colonies composed of undifferentiated hES cells as well as surrounding non-colony cells, human-ES-cell-derived fibroblast-like cells (hdFs). The hdFs were seen in both feeder and feeder-free hES cell culture and were found to predominantly express FGFR1, but not the hES cell marker Oct-4. Notably, quantitative real-time polymerase chain reaction (qPCR) analyses showed that $\mathrm{IGF} 1 \mathrm{R}^{+}$cell fraction was highly enriched for IGFIR and Oct-4 expression, whereas $F G F R 1$ transcript was unexpectedly limited to IGF1R cells, when hES cell cultures were sorted into $\mathrm{IGF}^{+} \mathrm{R}^{+}$and IGF1R- cells by flurorescence-activated cell sorting. Using immunocytochemistry, they further confirmed that IGF1R was expressed exclusively by Oct-4expressing colonies. In contrast, FGFR1 expression was restricted to hdFs. Although the tested hES cells from four independent lines (H1, H9, HES3 and CA1) all exhibited this distinct expression pattern [5], examination of more $\mathrm{hES}$ cell lines will provide more information about the 
niche of hES cells. The facts that FGFR1 did not co-localize with Oct-4-expressing undifferentiated hES cells and that FGFR1 transcript was restricted to hdFs clearly point out that bFGF does not sustain hES cell identity directly on hES cells, but indirectly on hdFs.

The next questions were how bFGF functions via hdFs and what the role of IGF-II is in the maintenance of hES identity. Bendall et al demonstrated that addition of IFG-II alone in defined conditions was sufficient to maintain hES pluripotent development potential and IGF1R blocking increased $\mathrm{hES}$ cell apoptosis, indicating that $\mathrm{hES}$ cells are dependent on external signals through IGF1R. On the other hand, the study revealed that inhibition of FGF receptor did not affect $h E S$ expansion, but induced $h E S$ cell differentiation, especially into the default path of neural differentiation, further confirming the essential effect of FGF on hES cell culture. They also found that conventional hES cell culture can produce adequate amounts of endogenous IGFII and exogenous bFGF greatly enhanced IGF-II expression in hdFs [5]. The findings are in agreement with the fact that bFGF functions in $\mathrm{hES}$ cell culture in feeder-free condition, where it does not act directly on hES cells, as previously predicted, but instead on hES cell-derived spontaneously differentiated cells, probably hdFs. Nevertheless, it would be interesting to learn whether IGF-II expression in hdFs is influenced if FGFR1 is interfered with. Currently we do not know if other elements other than FGFR1 can regulate IGF-II expression from hdFs. However, the observation by Bendall et al that inhibition of IGF1R and FGFR1 produces differential effects on hES cell culture highly supports this possibility. In addition to IGF, the authors identified TGF- $\beta$ as another factor regulating hES cell functions. Similar to FGFR1, TGF- $\beta$ was highly expressed by hdFs, but not by the IGF1R ${ }^{+}$cells, and inhibition of TGF- $\beta$ pathway induced hES cell differentiation, but did not affect the number of cells. The results suggest that TGF- $\beta$ produced by hdFs is required for hES cells to maintain an undifferentiated state.

Based on the findings described above, Bendall et al proposed a model to describe the interaction between hES cells and their niche, hdFs, where hES cells in culture autologously generate differentiated fibroblast-like niche cells (hdFs), which secret regulatory factors, including IGF-II and TGF- $\beta$, to support survival and self-renewal of hES cells by acting on receptors (e.g. IGF1R) present on hES cell membrane; production of IGF-II, TGF- $\beta$, and possibly other factors from hdFs is under the regulation of exogenous bFGF or endogenously produced FGFs via receptors (e.g. FGFR1) located on cell membrane of hdFs. Overall, the most significant advances made by this study include i) clarification of the indirect role of FGF in supporting $\mathrm{hES}$ cell culture, through acting on hdFs to prevent hES cell differentiation; ii) discovery of a central and direct role for the IGF-II/IGF1R pathway in regulating hES cell functions; iii) providing the first evidence that $\mathrm{hES}$ cells are capable of generating a stem cell niche in vitro. This niche contains not only a heterologous cell type, hdFs, but also paracrine factors, IGF-II and TGF- $\beta$. According to Scadden [3], it is possible that there are other unidentified components in the hES cell niche. The system provides us an excellent starting point from which to further explore the interaction between hES cells and their niche. The better definition of the niche will greatly facilitate the optimization of hES cell culture conditions.

\section{References}

1 Schofield R. The relationship between the spleen colony-forming cell and the haemopoietic stem cell. Blood Cells 1978; 4:7-25.

2 Li L, Xie T. Stem cell niche: structure and function. Annu Rev Cell Dev Biol 2005; 21: 605-631.

3 Scadden DT. The stem-cell niche as an entity of action. Nature 2006; 441:1075-1079.

4 Perry JM, Li L. Disrupting the stem cell niche: good seeds in bad soil. Cell 2007; 129:1045-1047.

5 Bendall SC, Stewart MH, Menendez P, et al. IGF and FGF cooperatively establish the regulatory stem cell niche of pluripotent human cells in vitro. Nature doi:10.1038/nature06027.

6 Thomson JA, Itskovitz-Eldor J, Shapiro SS, et al. Embryonic stem cell lines derived from human blastocysts. Science 1998; 282:1145-1147.

7 Ludwig TE, Levenstein ME, Jones JM, et al. Derivation of human embryonic stem cells in defined conditions. Nat Biotechnol 2006; 24:185-187.

8 DeChiara TM, Efstratiadis A, Robertson EJ. A growth-deficiency phenotype in heterozygous mice carrying an insulin-like growth factor II gene disrupted by targeting. Nature 1990; 345: 78-80.

9 Levenstein ME, Ludwig TE, Xu RH, et al. Basic fibroblast growth factor support of human embryonic stem cell self-renewal. Stem Cells 2006; 24:568-574. 